\title{
Parturient satisfaction with labour epidural and factors affecting satisfaction scores: experiences from a tertiary centre in Southern India
}

\author{
Leah Raju George ${ }^{1}$, Thenmozhi Mani², Ekta Rai ${ }^{1 *}$
}

${ }^{1}$ Department of Anaesthesia, ${ }^{2}$ Department of Biostatistics, Christian Medical College and Hospital, Vellore, Tamil Nadu, India

Received: 16 November 2019

Accepted: 10 December 2019

*Correspondence:

Dr. Ekta Rai,

E-mail: ektarai@yahoo.com

Copyright: ( $)$ the author(s), publisher and licensee Medip Academy. This is an open-access article distributed under the terms of the Creative Commons Attribution Non-Commercial License, which permits unrestricted non-commercial use, distribution, and reproduction in any medium, provided the original work is properly cited.

\begin{abstract}
Background: Labour analgesia though widely practised is still not routinely administered to all parturients in India. We conducted this historical observational cross-sectional study to assess parturient knowledge and factors affecting satisfaction in those who received epidural labour analgesia; aiming at improving the services.

Methods: All parturients requesting epidural labour analgesia have the catheter sited and the drug administered as per institutional protocols. A feedback form is given to these parturients, postnatally. The form consists of questions regarding her knowledge of and experience with labour analgesia, including her satisfaction score on a scale of 0 to 10. Author collected these forms and analyzed them along with information from the labour epidural register and the discharge summary.

Results: The mean satisfaction score was 7.1 (SD- 2.28). 46 parturients (31.08\%) had 'low satisfaction' ( $<7$ score) and 102 parturients $(68.9 \%)$ had 'high satisfaction' $(\geq 7$ score). The timing of initiation of epidural analgesia within the parturients expectations and adequate analgesia were two factors that were found to affect satisfaction scores, with statistically significant values $(\mathrm{p}=0.002$ and $\mathrm{p}=0.006$ respectively). Those with a very short or very long duration of labour analgesia, were more likely to give less satisfaction scores $(\mathrm{p}=0.023$ and $\mathrm{p}=0.002)$. Only $30 \%$ of parturients had heard of labour analgesia in the past.

Conclusions: In this setting good analgesia and receiving it on time are of utmost importance in those receiving epidural labor analgesia, emphasising the need for adequate analgesia and prompt initiation of the same.
\end{abstract}

Keywords: Epidural analgesia, Labour analgesia, Patient satisfaction, Satisfaction scores

\section{INTRODUCTION}

Labour analgesia has come a long way since it was first administered by James Young Simpson in $1847 .{ }^{1}$ Labour epidural is widely practised all over the world, and today in many developed countries the availability of labour analgesia services, primarily the epidural analgesia reflects standard obstetric care. Although it is beyond its infancy in the Indian scenario; it is still not routinely administered or offered to all parturients owing to low availability, monetary reasons and lack of knowledge amongst parturients. Institution has been providing labour epidural services for the past three decades. Author wanted to understand the patients' experiences with the epidural services.

There are only few studies done regarding the Indian patient's expectations during labour and factors affecting their satisfaction, and even fewer evaluating satisfaction with epidural. ${ }^{2-5}$ The aim of this study is to determine 
patient knowledge and the factors affecting parturient satisfaction with labour epidural thereby aiding us in improving the services.

\section{METHODS}

This is a historical, observational cross- sectional study of parturients who had epidural analgesia for labour in the year 2017.

The routine practice in the institution is that the parturient is counselled by the anesthetists in the labour room, regarding the availability of labour analgesia services. Those who agree and consent for the same have it sited once they are in active labour, in discussion with the obstetrician. The epidural is placed by conventional methods by a consultant or if a postgraduate student, under a consultant's supervision. Titrated volumes of $0.1 \%$ ropivacaine with $10-20 \mathrm{mcg}$ of fentanyl is used for the initial bolus followed by $0.1 \%$ ropivacaine plus 2 $\mathrm{mcg} / \mathrm{cc}$ of Fentanyl as the continuous infusion. Postnatally after the catheter is removed and the patient is shifted to the ward, an anaesthetist different from the team that sited the catheter administers a feedback form formulated by the department with questions assessing her prior knowledge about labour analgesia and her current experience with the same. Patients enter their overall satisfaction scores regarding the anaesthetic services and the efficacy of the labour epidural on a range of $0-10$. The anaesthesia department also maintains an epidural register which documents details such as time of insertion of catheter and experience of the anesthesiologist.

The historic data was collected from the feedback forms, the epidural register and the electronic discharge summary.

\section{Inclusion criteria}

All patients who had an epidural inserted for labour analgesia in the year 2017 .

\section{Exclusion criteria}

Patients whose forms had incomplete data like missing or wrong hospital number, for which further information could not be collected.

There are many studies that state that analgesia is not the only contributor to patient satisfaction. ${ }^{2,6-9}$ These are the various factors that were analysed, that may have an association with satisfaction in the society.

- Factors related to her past obstetric history such as history of abortions or treatment for infertility

- Factors related to the epidural like experience of the anesthesiologist who placed it, whether analgesia was initiated promptly and whether there were any epidural related complications.
- Factors related to her labour and delivery- mode of delivery, presence/ degree of perineal tear, time between placement of epidural catheter and deliverywhich it was termed as duration of epidural analgesia

- Factors related to the baby- the sex of the child, the Apgar score and nursery admission

- Factors related to the immediate post-natal periodfever on day one.

Neural deficit was defined as numbness and or weakness unilaterally or bilaterally persisting 24 hours after delivery. Urinary retention was defined as the inability to void spontaneously after delivery or a post void residue of more than $150 \mathrm{cc}$ after micturition, requiring an indwelling urinary catheter for 48 hours. ${ }^{10}$

\section{Statistical analysis}

Data was collated into Microsoft Excel and analysed in SPSS version 21. The Institutional Review Board of the centre approved of the study. (Ref: 11495 [Retro]).

Descriptive statistics such as mean, standard deviation, median and inter quartile range was used. For categorical variables number and proportion was used. Chi-square test was done to test the association between potential covariates and level of patient's satisfaction. The level of patient's satisfaction score was categorized into 'low satisfaction' $(<7)$ and 'high satisfaction' $(\geq 7)$. Association between continuous potential covariates and level of patient's satisfaction (low/high satisfaction) was assessed using Independent student's t-test for normally distributed potential covariates and Mann-Whitney U test was used for skewed data. The association from the logistic regression models were characterized using odds ratio (OR) and corresponding 95\% confidence interval $(95 \%$ CI). Stratified analysis was done for those who received epidural on time and for overall pain relief with level of patient's satisfaction (low/high satisfaction). Adjusted odds ratio was calculated based on MantelHaenszel test. P-value less than 0.05 were considered as statistically significant.

\section{RESULTS}

The details of 228 parturients who receive labour epidural during this period were entered and analysed. 193 $(84.6 \%)$ were primigravids and 35 (15.4\%) were multigravidas. 67 parturients $(29.8 \%)$ had heard of labour analgesia before. $201(90.1 \%)$ felt adequate information was provided by the anaesthesiologist regarding the epidural and $198(88 \%)$ felt they had enough time to consent for the same. $194(86.2 \%)$ felt they received the epidural 'on time'. 'On time' was a subjective assessment by the parturient of promptness of initiation of epidural analgesia, once she requested the services. $146(68.9 \%)$ said the infusion was given continuously through her labour. With regards to analgesia $190(85.6 \%)$ felt their overall pain relief was adequate. Author assessed pain further in terms of when the pain was present and 
intensity of pain and found that $60 \%$ of parturients had no pain or the pain was present only sometimes. $25 \%$ had pain only during second stage. $60 \%$ of parturients had no pain or mild pain. This is illustrated in the graphs in Figure 1 and 2. $192(85.7 \%)$ parturients said they would recommend labour epidural to their friends and relatives. $148(66.7 \%)$ said they would request an epidural for their next pregnancy. $17(7.6 \%)$ said they were not sure if they wanted an epidural for the next pregnancy, 9 (4\%) said they had completed their family with this delivery and 48 $(21.6 \%)$ said they would not request an epidural in their next pregnancy.

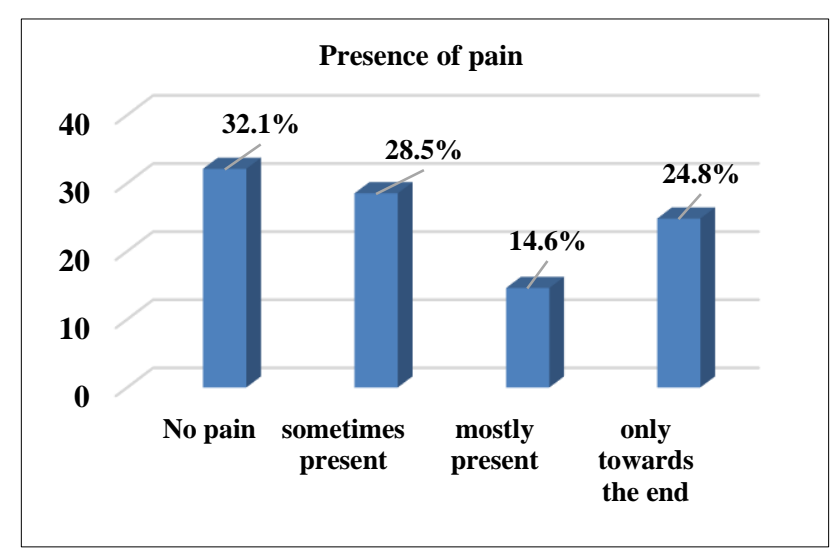

Figure 1: Timing of the presence of pain while on epidural labour analgesia.

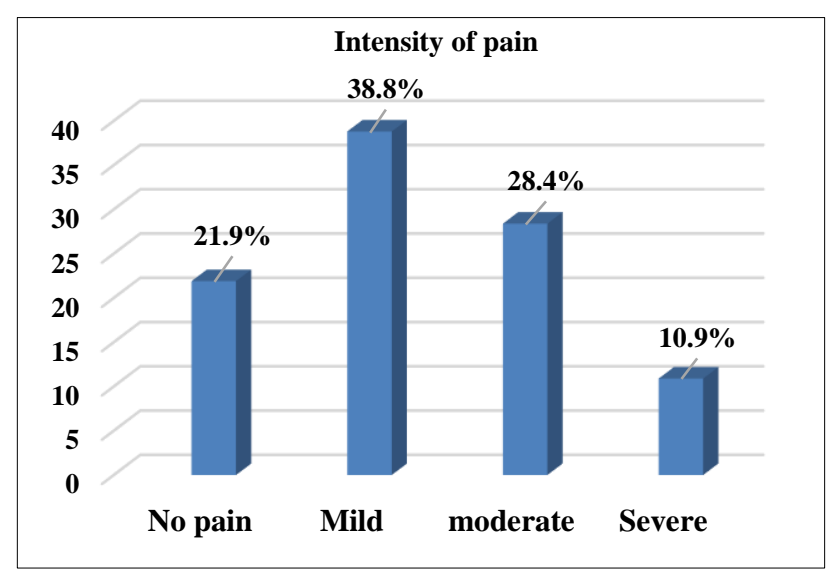

Figure 2: Intensity of pain while on epidural labour analgesia.

It was found that $156(69.6 \%)$ parturients delivered vaginally and 68 (30.4\%) delivered by LSCS (lower section caesarean section). This is further explained in Figure 3.

There were some patients who could not comprehend the concept of a satisfaction score and hence could not put a number to it. These patients were excluded from further analysis. 148 patients entered their overall satisfaction score and it was assessed the factors affecting satisfaction in these patients. The mean satisfaction score was 7.1 (SD- 2.28). Author considered less than 7 as 'low satisfaction' and more than or equal to 7 as 'high satisfaction'. 46 parturients (31.08\%) had 'low satisfaction' and 102 parturients $(68.9 \%)$ had 'high satisfaction'.

Table 1: Unadjusted logistic regression analysis of potential covariates of patient dissatisfaction. Status 'high satisfaction' was used as the reference category.

\begin{tabular}{|c|c|c|c|}
\hline Variable & OR & $95 \% \mathrm{CI}$ & P-value \\
\hline \multicolumn{4}{|c|}{ History of abortion /infertility } \\
\hline No & 1.00 & \multirow{2}{*}{$0.32-1.65$} & \multirow{2}{*}{0.443} \\
\hline Yes & 0.72 & & \\
\hline \multicolumn{4}{|l|}{ First pregnancy } \\
\hline Yes & 1.00 & \multirow{2}{*}{$1.29-8.20$} & \multirow{2}{*}{0.013} \\
\hline No & 3.25 & & \\
\hline \multicolumn{4}{|c|}{ Heard of labour analgesia services before } \\
\hline Yes & 1.00 & \multirow{2}{*}{$0.38-1.69$} & \multirow{2}{*}{0.564} \\
\hline No & 0.80 & & \\
\hline \multicolumn{4}{|c|}{ Adequate information regarding epidural given } \\
\hline Yes & 1.00 & \multirow{2}{*}{$0.93-8.08$} & \multirow{2}{*}{0.068} \\
\hline No & 2.74 & & \\
\hline \multicolumn{4}{|c|}{ Epidural received on time } \\
\hline Yes & 1.00 & \multirow{2}{*}{$1.79-12.43$} & \multirow{2}{*}{0.002} \\
\hline No & 4.72 & & \\
\hline \multicolumn{4}{|c|}{ Experience of anaesthetist } \\
\hline Consultant & 1.00 & \multirow{2}{*}{$0.49-2.36$} & \multirow{2}{*}{0.863} \\
\hline Post graduate & 1.07 & & \\
\hline \multicolumn{4}{|l|}{ Overall pain relief } \\
\hline Yes & 1.00 & \multirow{2}{*}{$1.50-11.06$} & \multirow{2}{*}{0.006} \\
\hline No & 4.08 & & \\
\hline \multicolumn{4}{|c|}{$\begin{array}{l}\text { Time between placement of epidural catheter and } \\
\text { delivery }\end{array}$} \\
\hline 121 to 600 minutes & 1.00 & & \\
\hline$<120$ minutes & 5.69 & $1.27-25.57$ & 0.023 \\
\hline$>600$ minutes & 3.99 & $1.63-9.76$ & 0.002 \\
\hline \multicolumn{4}{|c|}{ Epidural related complications } \\
\hline No & 1.00 & \multirow{2}{*}{$0.23-1.91$} & \multirow{2}{*}{0.439} \\
\hline Yes & 0.65 & & \\
\hline \multicolumn{4}{|l|}{ Mode of delivery } \\
\hline Vaginal & 1.00 & \multirow{2}{*}{$0.74-3.31$} & \multirow{2}{*}{0.248} \\
\hline LSCS & 1.56 & & \\
\hline \multicolumn{4}{|l|}{ Sex of the child } \\
\hline Male & 1.00 & \multirow{2}{*}{$0.62-2.55$} & 517 \\
\hline Female & 1.26 & & 0.311 \\
\hline
\end{tabular}

The unadjusted logistic regression analysis of these results is presented in Table 1. Multigravidas had 3.25 (95\% CI: $1.29-8.20)$ times the odds of having a low satisfaction as compared to primigravids, which was statistically significant $(\mathrm{p}=0.013)$. Those who did not receive the epidural 'on time' had nearly 5 (95\% CI: 1.79-12.43) times the odds of low satisfaction as compared to those who received the epidural on time, which was statistically significant $(\mathrm{p}=0.002)$. Those who did not have overall pain relief had 4 (95\% CI: $1.50-$ 11.06) times the odds of low satisfaction as compared to 
those who had overall pain relief, which was statistically significant $(\mathrm{p}=0.006)$. Those who had a very short duration of epidural analgesia $\leq 120$ minutes had $6(95 \%$ CI: $1.27-25.57)$ times the odds of low satisfaction and those with $>600$ minutes duration of epidural analgesia had nearly 4 (95\% CI: 1.63-9.76) times the odds of low satisfaction as compared to women who had 121 to 600 minutes of epidural analgesia, which was also statistically significant $(\mathrm{p}=0.023$ and $\mathrm{p}=0.002)$.

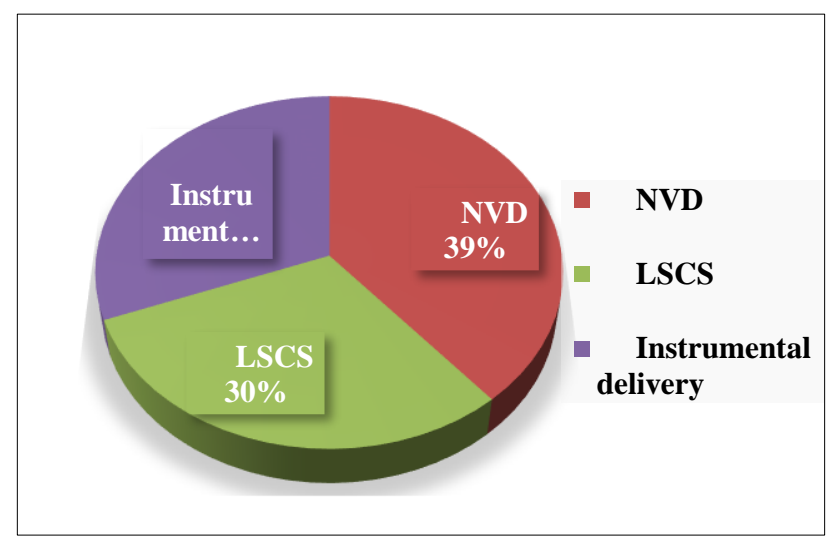

Figure 3: Mode of delivery.

\section{LSCS- lower section caesarean section}

Author did a stratified analysis for epidural being received 'on time' with overall pain relief. It was found that in those who received it 'on time', those with good pain relief were 2.5 times more likely to be satisfied (95\% CI: 0.79 - 7.86) as compared to those without good pain relief, which was not found to be statistically significant $(\mathrm{p}=0.120)$. In those who did not receive it 'on time', those with good pain relief had 9 times the odds (95\% CI: 0.85 - 94.90) of higher satisfaction as compared to those without pain relief, which was not statistically significant $(\mathrm{p}=0.068)$. After adjusting for the epidural being received on time, those who had overall pain relief had 3.32 times the odds of having higher satisfaction as compared to those who did not have overall pain relief, and this was found to be statistically significant $(\mathrm{p}=$ 0.017). Looking for an interaction between the two it was found that there was a much higher level of satisfaction with an odds ratio of 19.92 indicating an interaction between timeliness and good analgesia and that was found to be statistically significant ( $\mathrm{p}-0.007)$.

\section{DISCUSSION}

Though initially condemned by religious bodies; once administered by John Snow to Britain's Queen Victoria in 1853, labour analgesia gained popularity. After having gone through a period known as the "dark ages of obstetric anaestheisia" between the 1930 s to the 40s, vast improvements in the safety of regional analgesia were made in the 1950 s and advances continue to be made. ${ }^{11,12}$
Studies from India on parturient's experience, satisfaction and factors affecting the same are but few, looking into various aspects like perceptions, expectations and attitudes. A study from Southern India concluded that analgesia is not always of primary importance but meeting the parturient's expectations plays a major role in parturient satisfaction. ${ }^{2}$ Kamakshi et al conducted a study in a tertiary centre in North West India, and highlighted that parturient knowledge is poor and there is a wide gap between the desire for analgesia and the availability of prompt services. ${ }^{4}$ Samanta et al found better maternal satisfaction and analgesic efficacy with epidural compared to intramuscular Tramadol which is the readily available analgesic in most settings. ${ }^{5}$ A study based on individual in depth interviews and focus group discussions of women who delivered at home or in the primary health centres of rural East India found that the expectations of parturients in that set up was; affordable and accessible health care services, along with privacy and emotional support. ${ }^{3}$

Institution is a tertiary to quaternary care centre catering to a mixed population of rural and urban patients. Author conducted 14,696 deliveries in 2017; with the rates of normal vaginal deliveries being $50.7 \%$, instrumental $15.98 \%$ and caesarean $30.72 \%$.

Various parameters were studied and their effects on satisfaction scores amongst those who received labour epidural.

\section{Prior knowledge and information provided about labour analgesia}

In the culture, especially amongst the less educated, often decisions regarding labour analgesia are taken by the family rather than the parturient. $70.2 \%$ of parturients had not heard of labour analgesia. Current practise is that the anaesthetist counsels parturients in the labour room. Though occasionally discussed in the antenatal period it is not routinely addressed. Being a high-volume centre, the obstetricians and anaesthetists are hard pressed for time, highlighting the need for us to set up a nurse-based clinic for antenatal patients explaining the options and clarifying queries and misconceptions of labour analgesia. Patient satisfaction was not affected by prior knowledge of epidural analgesia (p-0.567). Once the anaesthetists counselled parturients, majority; $90 \%$ felt adequate information was given regarding the epidural and that they had enough time to consent for the same. This too did not impact patient satisfaction (p-0.068).

Once the parturient availed of the services most $(85.7 \%)$ were happy to recommend it to their friends or relatives. $66.7 \%$ said they would request an epidural for their next pregnancy, while $7.6 \%$ said they were not sure if they wanted an epidural for the next pregnancy, saying they were willing to experience the pain the next time and request an epidural if the pain became intolerable. $4 \%$ said they had completed their family with this delivery 
and $21.6 \%$ said they would not request an epidural in their next pregnancy.

\section{Promptness and efficacy of analgesia}

In the scenario where awareness and the demand are low, it was found that if pain relief is good satisfaction is high and secondly if prompt pain relief is provided, satisfaction is high. Once it was put together, author got a much higher level of satisfaction with an odds ratio of 19.92, indicating an interaction between these two variables that was statistically significant (p-0.007). Thus, it can be concluded that the two main elements of poor satisfaction are either poor pain relief or untimely placement of the catheter. If the patient received the epidural too late into her progress of labour she had already been through maximal pain and despite adequate analgesia gave a low score, emphasising the need to insert the epidural at maternal request as per practise guidelines by the American Society of Anaesthesiologists (ASA) and American College of Obstetricians and Gynaecologists (ACOG) or within 30 minutes of the patient's requests as per the NICE guidelines and RCOA audit standards. ${ }^{13-16}$ Thus the obstetricians plays an important role in informing the anaesthetist early, as soon as the parturient requests the epidural.

\section{Parity}

$84.4 \%$ of the patients were primigravida. There are other studies too that found higher request rates amongst primigravids. ${ }^{17,18}$ The primigravid is more likely to request for labour analgesia, owing to a greater fear of labour pains. Multigravidas were three times more likely to give lower satisfaction scores, (p-0.013) in keeping with the study done by Tan et al, in Singapore. ${ }^{18}$ A likely explanation of this finding is that a multiparous parturient probably has higher expectations of analgesia.

\section{Duration of epidural analgesia}

Parturients who had a very short duration ( $<120 \mathrm{~min})$ of epidural analgesia had lower satisfaction ( $\mathrm{p}-0.023)$. There are two reasons for this; firstly, these parturients had already progressed through most part of her labour having been through most of the pain and secondly some of these parturients could have belonged to the group who wanted a natural delivery and then requested the epidural being unable to withstand the pain.

Kannan et al too interestingly found that those who expected natural delivery but were unable to fulfil this expectation were found to be less satisfied with their childbirth experience. ${ }^{8}$ Those who had an exceptionally long duration (>600 minutes) between the epidural insertion and delivery too had low satisfaction scores (p0.002). In these patients the duration of labour itself was long, exhaustion was more, they probably had an instrumental delivery or an LSCS and the chances of epidural catheter displacement and patchy block towards the end were probably higher and hence a multifactorial cause for dissatisfaction.

\section{Mode of delivery}

Mode of delivery did not affect satisfaction scores, though there were 16 patients who strongly attributed their caesarean section to the epidural.

\section{Gender}

While many states in India still battle with skewed child sex ratios, because of a preference for the male child, we looked to see if satisfaction scores were affected by sex of the child. ${ }^{19}$ It is interesting to find no statistically significant relation $(\mathrm{p}=0.517)$.

\section{Complications}

Only 13 patients had epidural related complications, 23 had neonatal complications, 17 had fever on the first post-operative day, and none had third degree perineal tear or Apgar <6. Author did not get statistically significant values to prove these as causes of dissatisfaction, this may be due to small numbers of the same.

In the Western world, multiple choices of analgesia are available, awareness is higher and thus the request rate is high. The parturient has greater autonomy in choosing her mode of analgesia. Besides analgesia the relationship between the caregiver and the parturient, her involvement in decision making and meeting her expectations are important factors that affect parturient satisfaction. ${ }^{7,9}$ In some studies when these factors were met there was higher satisfaction despite proven lesser analgesia with methods such as nitrous oxide and patient controlled remifentanil or when maternal expectations of natural birth methods were met. ${ }^{6,8,20}$ The disparity in the availability of health services in India is still a reality. In the semi urban to urban set up although $70 \%$ of the patients had not even heard of epidural analgesia, once they experienced quality care, they were happy to recommend it to others highlighting the need for us to educate the patients and provide them with a pleasant childbirth experience.

There are numerous aspects that can affect patient satisfaction. There are but a few studies were a validated questionnaire has been used to assess the same. ${ }^{21-23} \mathrm{~A}$ limitation of this study is that a validated questionnaire for feedback was not used and the sample size was low. Author need to plan towards a prospective study with a validated questionnaire once antenatal education, which is in its inception now is well in place.

\section{CONCLUSION}

Patient satisfaction with regards to labour epidural is multifactorial. In patients opting for epidural analgesia, 
prompt initiation and effective pain relief is of utmost importance. Author need to concentrate on antenatal counselling of patients to disseminate appropriate and accurate information regarding labour analgesia and providing timely and quality service to the patients.

\section{ACKNOWLEDGMENTS}

Authors would like to thank Dr Jayaprakash Muliyil for his expert advice on statistics and thank Miss Sajini B and $\mathrm{Mr}$ Edward Jonathan for their help with data collection.

\section{Funding: No funding sources}

Conflict of interest: None declared

Ethical approval: The study was approved by the Institutional Ethics Committee

\section{REFERENCES}

1. Dunn PM. Sir James Young Simpson (1811-1870) and obstetric anaesthesia. Arch Dis Child - Fetal Neonatal Ed. 2002;86(3):F207-9.

2. Bhatt H, Pandya S, Kolar G, Nirmalan PK. The impact of labour epidural analgesia on the childbirth expectation and experience at a tertiary care center in Southern India. J Clin Diagn Res JCDR. 2014;8(3):73-6.

3. Bhattacharyya S, Srivastava A, Avan BI. Delivery should happen soon and my pain will be reduced: understanding women's perception of good delivery care in India. Glob Health Action. 2013;6:22635.

4. Kamakshi G, Anju G, Tania S, Priyanka G, Kamya $\mathrm{B}$, Gegal P, et al. Epidural analgesia during labor: attitudes among expectant mothers and their care providers. Anesth Essays Res. 2018;12(2):501-5.

5. Samanta S, Jain K, Bhardwaj N, Jain V, Samanta S, Saha R. Maternal and foetal outcome after epidural labour analgesia in high-risk pregnancies. Indian $\mathrm{J}$ Anaesth. 2016;60(2):115.

6. Richardson MG, Lopez BM, Baysinger CL, Shotwell MS, Chestnut DH. Nitrous oxide during labor: maternal satisfaction does not depend exclusively on analgesic effectiveness. Anesth Analg. 2017;124(2):548-53.

7. Hodnett ED. Pain and women's satisfaction with the experience of childbirth: a systematic review. Am J Obstet Gynecol. 2002;186(5 Suppl Nature):S160172.

8. Kannan S, Jamison RN, Datta S. Maternal satisfaction and pain control in women electing natural childbirth. Reg Anesth Pain Med. 2001;26(5):468-72.

9. Camann W. Pain, pain relief, satisfaction and excellence in obstetric anesthesia: a surprisingly complex relationship. Anesth Analg. 2017;124(2):383.
10. Kekre AN, Vijayanand S, Dasgupta R, Kekre N. Postpartum urinary retention after vaginal delivery. Int J Gynecol Obstet. 2011;112(2):112-5.

11. Gogarten W, Aken HV. A century of regional analgesia in obstetrics. Anesth Analg. 2000;91(4):773-5.

12. Gupta S, Partani S. Neuraxial techniques of labour analgesia. Indian J Anaesth. 2018;62(9):658.

13. Practice Guidelines for Obstetric Anesthesia. An updated report by the American Society of anesthesiologists task force on obstetric anesthesia and the society for obstetric anesthesia and perinatology. Anesthesiol J Am Soc Anesthesiol. 2016;124(2):270-300.

14. Practice Bulletin No. 177: obstetric analgesia and anesthesia. Obstet Gynecol. 2017;129(4):e73.

15. Royal College of Anaesthetists. Raising the Standard: a Compendium of Audit Recipes. 3rd Edition 2012. London: RCoA, 2012

16. CSQ-ARB2012-SEC8.pdf, 2012. Available at: https://www.salg.ac.uk/system/files/CSQ-ARB2012SEC8.pdf.

17. Koteles J, de Vrijer B, Penava D, Xie B. Maternal characteristics and satisfaction associated with intrapartum epidural analgesia use in Canadian women. Int J Obstet Anesth. 2012;21(4):317-23.

18. Tan DJA, Sultana R, Han NLR, Sia ATH, Sng BL. Investigating determinants for patient satisfaction in women receiving epidural analgesia for labour pain: a retrospective cohort study. BMC Anesthesiol. 2018;18(1):50.

19. Female foeticide in India. UNICEF. Available at: http://www.unicef.in/PressReleases/227/Femalefoeticide-in-India.

20. Frauenfelder S, Rijn R van, Radder CM, Vries MC de, Dijksman LM, Godfried MB. Patient satisfaction between remifentanil patient-controlled analgesia and epidural analgesia for labor pain. Acta Obstet Gynecol Scand. 2015;94(9):1014-21.

21. Morgan PJ, Halpern S, Lam-McCulloch J. Comparison of maternal satisfaction between epidural and spinal anesthesia for elective Cesarean section. Can J Anesth. 2000;47(10):956.

22. Sindhvananda W, Leelanukrom R, Rodanant O, Sriprajittichai P. Maternal satisfaction to epidural and spinal anesthesia for cesarean section. J Med Assoc Thailand. 2004;87(6):628-35.

23. Nikkola E, Läärä A, Hinkka S, Ekblad U, Kero P, Salonen M. Patient-controlled epidural analgesia in labor does not always improve maternal satisfaction. Acta Obstet Gynecol Scand. 2006;85(2):188-94.

Cite this article as: George LR, Mani T, Rai E. Parturient satisfaction with labour epidural and factors affecting satisfaction scores: experiences from a tertiary centre in Southern India. Int J Reprod Contracept Obstet Gynecol 2020;9:383-8. 